\title{
Asociación entre síntomas depresivos de las madres y control metabólico en adolescentes con Diabetes Mellitus tipo 1
}

\author{
Association between depressive symptoms in mothers and metabolic control \\ in adolescents with type 1 diabetes
}

\author{
Denise von Borries ${ }^{\mathrm{a}}$, Patricio Astudillo ${ }^{\mathrm{b}}$, Viviana Pérez ${ }^{\mathrm{c}}$, \\ Hernán García F. ${ }^{\mathrm{d}}$, Karime Rumie ${ }^{\mathrm{e}, \mathrm{f}}$, Hernán García B. ${ }^{\mathrm{e}}$
}

\author{
aDivisión de Pediatría, Escuela de Medicina, Pontificia Universidad Católica de Chile. Santiago, Chile \\ 'Departamento de Urgencia General, Clínica Alemana de Santiago, Chile \\ 'Enfermera, Programa de Diabetes tipo 1, Red de salud UC-Christus. Santiago, Chile \\ dHospital de Curanilahue, Servicio de Salud Arauco, Chile \\ eUnidad de Endocrinología Pediátrica, División de Pediatría, Escuela de Medicina, Pontificia Universidad Católica de Chile. Santiago, Chile \\ fUnidad de Endocrinología Pediátrica, Complejo Asistencial Doctor Sotero del Río, Servicio de Salud Metropolitano Sur Oriente. Santiago, Chile
}

Recibido: 27 de marzo de 2019; Aceptado: 4 de noviembre de 2019

¿Qué se sabe del tema que trata este estudio?

Los adolescentes con Diabetes Mellitus tipo 1 presentan peores controles metabólicos que otros grupos etarios, fenómeno con una explicación multifactorial. Algunos estudios han demostrado una asociación entre síntomas depresivos de las madres y peor control metabólico de sus hijos adolescente.
¿Qué aporta este estudio a lo ya conocido?

En nuestra población existe una alta prevalencia de síntomas depresivos en las madres de adolescentes con Diabetes Mellitus tipo 1 demostrándose también una asociación entre dichos síntomas y control metabólico en el adolescente.

\section{Resumen}

Un mal control metabólico en pacientes con Diabetes Mellitus tipo 1 (DM1) se asocia a complicaciones a corto y largo plazo. Los adolescentes con Diabetes tipo 1 presentan peor control metabólico comparado con pacientes de otros grupos etarios. Escasos estudios han demostrado una asociación entre síntomas depresivos de las madres con el control metabólico de sus hijos adolescente. Objetivo: Evaluar la asociación entre síntomas depresivos maternos y control metabólico de adolescentes con DM1. Sujetos y Método: Estudio observacional transversal realizado en adolescentes, edades 10 a 18 años, con diagnóstico de DM1 de más de un año de evolución y sus madres. Se aplicó test de Beck II, cuestionario de depresión infantil, cuestionario SALUFAM y cuestionario de datos sociodemográficos. Se realizó hemoglobina glicosilada capilar, como marcador de control metabólico. Resulta-
Palabras clave: Diabetes Mellitus tipo 1; síntomas depresivos; Hemoglobina glicosilada; adolescencia; madre 
dos: Se estudiaron 86 parejas (madre-hijo adolescente), adolescentes de edad media 14,04 años y 5,95 años de evolución de DM1. El 25,6\% (n 22) de las madres presentó síntomas depresivos, asociándose a peor control metabólico en sus hijos (HbAlc: 7.66\% y 8.91\%, p < 0,001). El 17,9\% de adolescentes presentó síntomas depresivos, no asociándose a síntomas depresivos maternos ni a peor control metabólico. Los síntomas depresivos maternos se asociaron a menor nivel educacional materno y paterno, mayor número de hijos en la familia, presencia de otros hermanos con enfermedades crónicas y a mayor vulnerabilidad en salud (SALUFAM). Conclusiones: La presencia de síntomas depresivos maternos se asocia a peor control metabólico en el adolescente con DM1, siendo fundamental un enfoque multidisciplinario familiar para obtener mejores resultados metabólicos en los adolescentes.

\begin{abstract}
Poor metabolic control in patients with Type 1 Diabetes Mellitus (T1DM) is associated with shortand long-term complications. Adolescents with T1DM present poorer metabolic control than patients of other age groups. Few studies have shown an association between mothers with depressive symptoms and the metabolic control of their adolescent children. Objective: To evaluate the association between maternal depressive symptoms and metabolic control of their adolescents with T1DM. Subjects and Method: Cross-sectional observational study carried out with adolescents aged between 10 and 18 years, with T1DM diagnosis of at least 1 year ago and their mothers. The Beck Depression Inventory-II and the SALUFAM questionnaire were applied, and sociodemographic data were collected. Glycosylated hemoglobin from capillary blood was used as a marker of metabolic control. Results: 86 couples (mother-adolescent children) were studied. The average age of the adolescents was 14.04 years and the average evolution time of T1DM was 5.95 years. $27.325 .6 \%$ of mothers had depressive symptoms, which was associated with worse metabolic control of their children (HbA1c of $7.66 \%$ and $8.91 \%$, p-value $<0.001$ ). $17.9 \%$ of adolescents had depressive symptoms, which was not associated with maternal depressive symptoms or worse metabolic control. Maternal depressive symptoms were also associated with lower maternal and paternal educational levels, high number of children in the family, presence of other siblings with chronic illnesses, and high health vulnerability (SALUFAM). Conclusions: The mother's depressive symptoms can be associated with worst metabolic control in T1MD adolescents. It is fundamental a multidisciplinary family approach to get better metabolic controls in T1DM adolescents.
\end{abstract}

\section{Keywords:}

Type 1 Diabetes Mellitus; Depressive Symptoms; Glycated Hemoglobin; Adolescence; Mother

\section{Introducción}

La Diabetes Mellitus tipo 1 (DM1) es una enfermedad metabólica crónica caracterizada por una hiperglicemia secundaria a una secreción insuficiente o nula de insulina por parte del páncreas. La incidencia varía según la población estudiada entre 0,5-52 por 100.000 habitantes/año. Durante las últimas décadas, se ha constatado a nivel mundial un aumento exponencial de casos de DM1, con un crecimiento anual de aproximadamente $3 \%{ }^{1}$, aumento que se ha demostrado también en Chile 2 .

El mal control metabólico en pacientes con DM1 se asocia a complicaciones micro y macrovasculares. La DM1 es un factor de riesgo cardiovascular, presentando un $15 \%$ de los pacientes algún evento cardiovascular en un seguimiento de 27 años $^{3}$. A mayor Hemoglobina Glicosilada (HbAlc), existe mayor frecuencia de eventos cardiovasculares, hospitalizaciones por cetoacidosis diabética ${ }^{4}$, vulnerabilidad en el desarrollo cerebral ${ }^{5}$, incidencia y severidad de complicacio- nes microvasculares, como retinopatía ${ }^{6-8}$, neuropatía y nefropatía diabética ${ }^{9}$, traduciéndose en una carga de enfermedad importante. Un mal control metabólico durante el periodo inicial de la enfermedad se asocia a mayor mortalidad, incluso en pacientes que consiguieron posteriormente $\mathrm{HbAlc}$ similares al resto, lo que se conoce como "memoria metabólica"10,11. Por lo tanto, mantener un buen control metabólico desde los primeros años de la enfermedad es fundamental.

La adolescencia es un momento crítico, siendo el periodo con peores controles metabólicos a nivel internacional ${ }^{12}$ y nacional, habiéndose reportado niveles de HbAlc sobre el objetivo (> 7,5\%) en $86 \%$ de los adolescentes chilenos entre 13 y 19 años con DM1 ${ }^{13}$. Esta situación responde a múltiples factores que se producen durante la adolescencia, tales como mayor independencia, influencias sociales, menor adherencia al tratamiento, patrones erráticos de alimentación y ejercicio, conductas de riesgo (consumo de alcohol y drogas), cambios hormonales propios de la pubertad y aumento de peso ${ }^{14}$. 
El diagnóstico de una enfermedad crónica, regímenes de tratamiento y controles demandantes, sumado a cambios de la dinámica familiar propios de la etapa de la adolescencia producen alteraciones en los patrones de supervisión, lo cual se traduce en dificultades en las relaciones interpersonales familiares ${ }^{15}$.

Aproximadamente el 20\% de padres de hijos con DM1 desarrolla trastorno depresivo mayor (TDM) y/o trastorno ansioso entre 1 a 4 años posterior al diagnóstico, siendo más prevalente que en la población gene$\mathrm{ral}^{15}$. Esto se asocia a mayor riesgo de hospitalización por complicaciones agudas de la diabetes ${ }^{16} \mathrm{y}$ riesgo de psicopatología en el adolescente ${ }^{15}$. Por otra parte, se ha demostrado que los síntomas depresivos son más frecuentes en pacientes pediátricos con DM1, comparado con población general ${ }^{17}$, asociándose a peor control metabólico ${ }^{18}$.

Otros factores que afectan los niveles de HbAlc en pacientes pediátricos son el nivel socioeconómico y educacional de los padres ${ }^{19}$, estado nutricional del paciente, adherencia al tratamiento, uso inyecciones comparado con microinfusores de insulina ${ }^{20}$, menor monitorización de glicemias ${ }^{12,21}$, uso de antipsicóticos ${ }^{22}$, menor conocimiento de los padres sobre la $D M 1^{23}$ y que los padres tengan miedo a la hipoglicemia $^{24}$.

Escasos estudios, todos realizados en población norteamericana, han demostrado asociación entre síntomas depresivos parentales con peor control metabólico en adolescentes con DM1 ${ }^{25-28}$. Los estudios han tratado de explicar la manera en que los síntomas depresivos parentales afectan de manera directa o indirecta el control metabólico. El estudio de Mackey et al. ${ }^{25}$ demuestra que los síntomas depresivos disminuirían la monitorización del adolescente, lo que se traduciría en menor adherencia y secundariamente peor control metabólico. Por su parte, Eckshtain et al. ${ }^{27}$ documenta que cuidadores con TDM presentarían involucramiento excesivo en el cuidado del adolescente, produciendo mayores conflictos intrafamiliares. Se plantea que los síntomas depresivos parentales afectan el control metabólico, incluso en ausencia de síntomas depresivos en el adolescente, por lo que tendría un efecto independiente a éstos.

Las guías disponibles de manejo de DM1 en pacientes pediátricos ${ }^{29-31}$ proponen un enfrentamiento integral del niño y su familia, con un enfoque en educación e intervenciones familiares para optimizar la cohesión y relaciones. Sin embargo, no incluyen tamizaje de síntomas depresivos parentales.

A la fecha no existen estudios en Latinoamérica, pudiendo constituir el reconocimiento de psicopatología en padres una oportunidad de intervención que contribuya a mejorar los alarmantes resultados de $\mathrm{HbA1c}$ observados en adolescentes con DM1 en nues- tro medio. El objetivo de esta investigación es analizar la asociación entre síntomas depresivos maternos y el control metabólico en adolescentes chilenos con DM1. Como objetivos secundarios se propuso establecer las prevalencias de síntomas depresivos en adolescentes con DM1 y sus madres, evaluar factores asociados a depresión materna, determinar la asociación entre síntomas depresivos maternos y en sus hijos y la asociación entre síntomas depresivos en éstos últimos y control metabólico.

\section{Sujetos y Método}

\section{Sujetos}

Estudio observacional transversal. Se reclutaron adolescentes entre 10 y 17 años, 11 meses 29 días, con diagnóstico DM1 de más de un año de evolución, en tratamiento en un centro terciario público y uno privado de la Región Metropolitana y sus madres. Se excluyeron aquellos adolescentes o madres con diagnóstico de otro trastorno psiquiátrico, que no corresponda a TDM o trastorno ansioso, diagnóstico en la madre y/o en el adolescente de discapacidad intelectual que no permita responder la encuesta, adolescente con comorbilidad severa (cardiopatía compleja, parálisis cerebral, cáncer, uso crónico de corticoides orales). Se realizó una selección de pacientes por conveniencia, reclutándose aquellos que acudieron a control durante el periodo del estudio.

\section{Variables evaluadas}

Se aplicó a las madres el inventario de depresión de Beck II (BDI-II), el cual consiste en un cuestionario de autorreporte de síntomas depresivos validado en Chile para mayores de 13 años ${ }^{32}$. Para el resultado del BDI-II, se consideró con sospecha de depresión si valor $>13$, para privilegiar una alta sensibilidad como screening. Los puntos de corte para graduar la severidad son leve (14-19), moderado (20-28) y severo (29$63)^{33}$. Además, se aplicó el cuestionario SALUFAM ${ }^{34}$, instrumento desarrollado en Chile que mide acuerdo y apoyo familiar, y permite identificar familias de mayor vulnerabilidad en Salud. Se ha demostrado que el punto de corte promedio de 3,7 puntos discrimina a las familias con buena y mala evolución clínica en diversas condiciones de salud.

A los adolescentes se les aplicó el cuestionario de depresión infantil (CDI), test de autoreporte de síntomas depresivos, ampliamente utilizado en niños y adolescentes con DM1 a nivel mundial ${ }^{35}$, validado en Chile $^{36}$. Se usó puntaje de corte de 18 puntos, valor establecido en la estandarización chilena. Tanto el BDI-II como CDI hacen referencia a la presencia de síntomas depresivos en las dos semanas previas a la realización 
del cuestionario. Además, se completó un cuestionario con datos sociodemográficos y clínicos, para determinar factores asociados a síntomas depresivos maternos.

Como estimación del control metabólico se utilizó HbA1C, único examen validado para estimar la calidad del control metabólico en pacientes con DM1. Traduce los niveles de glicemias promedios durante los últimos tres meses. En este estudio se midió de manera simultánea a los cuestionarios HbAlc capilar (Alcnow+, ptsDiagnostic, USA) que posee buena correlación con HbAlc en sangre venosa ${ }^{37}$.

\section{Análisis estadísticos}

El análisis estadístico se realizó con SPSS V25.0 (NY, USA). Para la descripción de las variables numéricas se utilizaron promedios y desviación estándar (DE). Para variables numéricas se realizó test de U Mann-Whitney y para categóricas Test de Fisher de dos colas. Se compararon los promedios de HbAlc entre los grupos de madres con y sin sospecha de TDM a través de test de U Mann-Whitney. Se realizó test de Kruskal-Wallis y post test de Dunn para evaluar la HbA1c según gravedad de los síntomas depresivos.

Se realizó un análisis multivariado binario forward para ajuste de las variables (sexo, presencia de otra enfermedad crónica en el adolescente, nivel educacional de la madre, presencia de síntomas depresivos maternos y en el adolescente y vulnerabilidad en salud) para el outcome de hemoglobina glicosilada ( $\mathrm{HbAlc}$ ) mayor a 7,5. Se presentan los valores de odds ratio (OR) para los análisis multivariado con intervalo de confianza de $95 \%$. Se consideró significativo todo valor $\mathrm{p}<0,05$.

El estudio fue aprobado por el Comité de Ética en Investigación de la Escuela de Medicina de la Pontificia Universidad Católica de Chile (ID 170222001) y del Servicio de Salud Metropolitano Sur Oriente. Este trabajo fue realizado bajo la Declaración de principios de Helsinki. Las madres firmaron un consentimiento informado y los adolescentes un asentimiento informado. Las madres y adolescentes que puntuaron en los cuestionarios con sospecha de depresión, se las contactó y se hizo entrega de una interconsulta para ser evaluados en salud mental según su previsión de salud.

\section{Resultados}

Se reclutaron 86 diadas, de un total de 238 sujetos con criterios de inclusión, siendo los adolescentes 42 $(48,8 \%)$ mujeres, la edad media de 14,04 años y 5,95 años de evolución de la enfermedad. La edad media de las madres fue 43,01 años.

El resultado del cuestionario BDI-II concluyó que
22 madres $(25,6 \%)$ presentan síntomas depresivos, siendo $10,5 \%$ leves, $10,5 \%$ moderados y $4,6 \%$ severos. La comparación de las variables entre los grupos de madres con y sin síntomas depresivos muestra una asociación de síntomas depresivos maternos con mayor número de hijos, menor niveles educacional materno y paterno, presencia de otros hijos con enfermedades crónicas y mayor vulnerabilidad en salud $(\mathrm{p}<0,05)($ tabla 1$)$. El cuestionario CDI $(\mathrm{n}=84)$ evidenció 15 (17,9\%) adolescentes con síntomas depresivos, no asociándose con síntomas depresivos maternos $(\mathrm{p}=1)($ tabla 1).

Los niveles de HbAlc de los hijos de madres con y sin síntomas depresivos fue $8,91 \%( \pm 1,52)$ y $7,66 \%$ $( \pm 1,34)$ respectivamente, con diferencia significativa $(\mathrm{p}<0,001)$ (figura 1). Al desglosar según gravedad de los síntomas depresivos maternos, se mantiene la significancia para el grupo de síntomas moderados (figura 2). Los síntomas depresivos maternos se asocian además a mayor riesgo de hospitalización por descompensación aguda de DM1 (OR 4,31 [IC95\% 1,25-14,97]).

$\mathrm{Al}$ ajustar las variables en un modelo logístico multivariado para eliminar confundentes, los síntomas depresivos maternos se asocian a mal control metabólico en el adolescente (HbAlc > 7,5\%) con un OR ajustado de 6,23 (IC95\% 1,16-33,5). A su vez, la presencia de otra enfermedad crónica en el adolescente y la vulnerabilidad en salud mantienen su asociación con mal control metabólico una vez ajustado (OR ajustado $3.72(1,08-12,85)$ y $5,38(1,003-28,95)$ respectivamente) (tabla 2).

Finalmente, los niveles $\mathrm{HbAlc}$ promedios en el grupo de adolescentes con y sin síntomas depresivos fue de $8,07 \%$ y $7,91 \%$ respectivamente, sin existir una diferencia estadísticamente significativa $(p=0,71)$ (figura 3 ).

\section{Discusión}

Según el presente estudio $25.6 \%$ de las madres de adolescentes con DM1 presentan síntomas depresivos, mayor a lo descrito en la población general al comparar con resultados de la encuesta nacional de salud (ENS) 2016-2017 de Chile, en la cual el subgrupo de mujeres arrojó $21,7 \%$ de síntomas depresivos. Si bien la ENS utilizó otro cuestionario (CIDI Short Form), correspondía también a una herramienta de screening y no de diagnóstico de $\mathrm{TDM}^{36}$. Estos resultados son concordantes con experiencias en otras poblaciones en que se objetivaron mayores niveles de distrés emocional y síntomas depresivos al compararlas con población general ${ }^{15}$. El conocer los factores que se asocian con síntomas depresivos maternos (tabla 1) permite identificar a madres de mayor riesgo. 
Tabla 1. Datos clínicos y sociodemográficos según presencia de síntomas depresivos en las madres de adolescentes con DM1

\begin{tabular}{|c|c|c|c|}
\hline \multirow[t]{2}{*}{ Variable } & \multicolumn{2}{|c|}{ Pacientes, $\mathrm{N}^{\circ}(\%)^{\mathrm{a}}(\mathrm{N}=86)$} & \multirow[t]{2}{*}{ Valor $\mathrm{p}$} \\
\hline & $\begin{array}{c}\text { Madres con síntomas } \\
\text { depresivos } \\
(\mathrm{N}=22(25,6 \%))\end{array}$ & $\begin{array}{c}\text { Madres sin síntomas } \\
\text { depresivos } \\
(\mathrm{N}=64(74,4 \%))\end{array}$ & \\
\hline Sexo femenino & $11(50)$ & $31(48,4)$ & 1 \\
\hline Edad de adolescentes. Media (DS). Años & $14,4 \quad(2,53)$ & $13,9(2,22)$ & 0,42 \\
\hline Edad de madres. Media (DS). Años & $44,5 \quad(9,52)$ & $42,5(7,53)$ & 0,51 \\
\hline Tiempo evolución de DM1. Años & $5,03(3,74)$ & $6,27(3,81)$ & 0,157 \\
\hline $\begin{array}{l}\text { Educación maternab } \\
\text { Menos de } 12 \text { años } \\
12 \text { años } \\
\text { Estudio técnico } \\
\text { Estudio universitario }\end{array}$ & $\begin{array}{lr}4 & (18,2) \\
9 & (40,9) \\
8 & (36,4) \\
1 & (4,5)\end{array}$ & $\begin{array}{c}6(10) \\
18(30) \\
19(31,7) \\
17(28,3)\end{array}$ & $\begin{array}{l}0,99 \\
0,82 \\
\mathbf{0 , 0 2 *}\end{array}$ \\
\hline $\begin{array}{l}\text { Tipo trabajo materno } \\
\text { Trabajadora dependiente } \\
\text { Trabajadora independiente } \\
\text { Dueña de casa } \\
\text { Desempleada }\end{array}$ & $\begin{array}{rr}7 & (31,8) \\
5 & (22,7) \\
9 & (40,9) \\
1 & (4,5)\end{array}$ & $\begin{array}{rr}25 & (41,7) \\
13 & (21,7) \\
19 & (31,7) \\
3 & (5,0)\end{array}$ & 0,68 \\
\hline Madre con enfermedad crónicab & $10(45,5)$ & $27 \quad(45,0)$ & 1 \\
\hline Edad del padre. Media (DS). años & $41,6(12,83)$ & $43,6(7,8)$ & 0,43 \\
\hline $\begin{array}{l}\text { Educación paternac } \\
\text { Menos de } 12 \text { años } \\
12 \text { años } \\
\text { Estudio técnico } \\
\text { Estudio universitario }\end{array}$ & $\begin{array}{ll}6 & (30) \\
6 & (30) \\
6 & (30) \\
2 & (10)\end{array}$ & $\begin{array}{rr}2 & (3,8) \\
18 & (34,6) \\
15 & (28,8) \\
17 & (32,3)\end{array}$ & $\begin{array}{l}0,04 \text { * } \\
0,02 \text { * } \\
0,004 \text { * }\end{array}$ \\
\hline $\begin{array}{l}\text { Tipo trabajo paterno } \\
\text { Trabajador dependiente } \\
\text { Trabajador independiente } \\
\text { Dueño de casa } \\
\text { Desempleado }\end{array}$ & $\begin{array}{rr}12 & (60) \\
7 & (35) \\
0 & (0) \\
1 & (5)\end{array}$ & $\begin{aligned} 34 & (66,7) \\
14 & (27,5) \\
1 & (2) \\
2 & (3,9)\end{aligned}$ & 0,46 \\
\hline Número de hijos en la familia. Media (DS) & $3,45(1,06)$ & $2,19(1,19)$ & $<0,001 *$ \\
\hline Número de personas viviendo en la casa. Media (DS) & $4,5 \quad(1,14)$ & $4,06(1,55)$ & 0,11 \\
\hline Ingreso per-cápita. Media (DS) \$ & $123.101(95.684)$ & $322.514(439.180)$ & 0,099 \\
\hline Hermano con enfermedad crónicae & $7(33,3)$ & $6 \quad(10,3)$ & $0,03 *$ \\
\hline Otra enfermedad crónica en adolescente ${ }^{f}$ & $6(28,6)$ & $18(29,5)$ & 1 \\
\hline $\begin{array}{l}\text { Estado nutricional }^{\text {b }} \\
\text { Riesgo de desnutrición } \\
\text { Eutrofia } \\
\text { Sobrepeso } \\
\text { Obesidad }\end{array}$ & $\begin{array}{rr}1 & (4,5) \\
16 & (72,8) \\
4 & (18,2) \\
1 & (4,5)\end{array}$ & $\begin{array}{rr}2 & (3,3) \\
32 & (53,3) \\
19 & (31,7) \\
7 & (11,7)\end{array}$ & $\begin{array}{l}0,99 \\
0,26 \\
0,45\end{array}$ \\
\hline $\begin{array}{l}\text { Tipo de tratamiento } \\
\text { Basal/bolo } \\
\text { Microinfusor insulina }{ }^{\text {b }} \\
\text { Hospitalización por descompensación aguda de DM1 en último añog }\end{array}$ & $\begin{array}{c}0 \quad(0) \\
22(100 \%) \\
5(23,8)\end{array}$ & $\begin{array}{rc}4 & (6,7 \%) \\
56 & (93 \%) \\
4 & (6,8)\end{array}$ & $0,048 *$ \\
\hline Vulnerabilidad en salud según SALUFAM ${ }^{h}$ & $15(68,2)$ & $9 \quad(14,8)$ & $<0,001 *$ \\
\hline Síntomas depresivos en adolescentes ${ }^{i}$ & 4 (19) & $11(17,5)$ & 1 \\
\hline HbA1c capilar & $8,91(1,52)$ & $7,66(1,34)$ & $<0,001 *$ \\
\hline
\end{tabular}

DS: Desviación estándar, OR (Odds ratio), 95\% IC (Intervalo de confianza al 95\%), aData representa el No (\%) de pacientes, salvo especificado de otra manera; 'batos disponibles de 22 y 60 pacientes respectivamente; 'Datos disponibles de 20 y 52 pacientes respectivamente; ${ }^{\mathrm{d}}$ Datos disponibles de 20 y 51 pacientes respectivamente; eDatos disponibles de 21 y 58 pacientes respectivamente; ${ }^{f}$ Datos disponibles de 21 y 61 pacientes respectivamente; ${ }^{9}$ Datos disponibles de 21 y 59 pacientes respectivamente; 'Datos disponibles de 22 y 61 pacientes respectivamente; 'Datos disponibles de 21 y 63 pacientes respectivamente; 'valor $p$ no calculado porque una o más categorías contiene cero. *Significativo. 


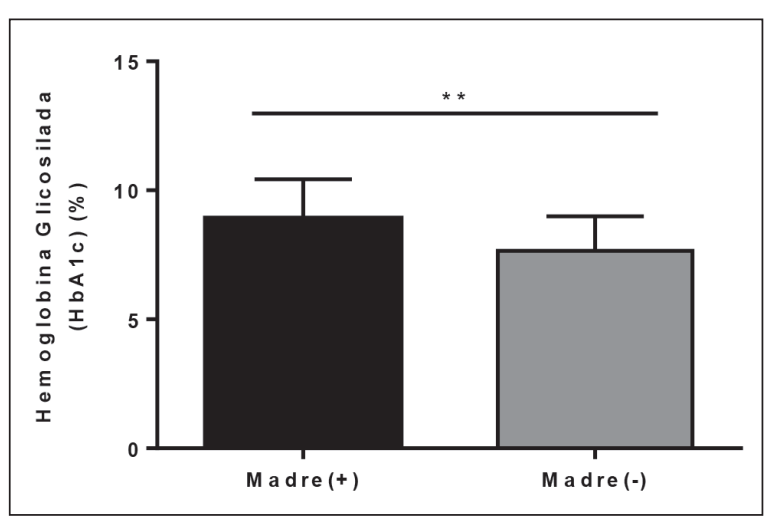

Figura 1. $\mathrm{HbA} 1 \mathrm{c}$ promedio en pacientes con madre con $(+)$ y $\sin$ (-) síntomas depresivos. Test de U Mann-Whitney, ${ }^{* *} p<0,001$.

El presente estudio demuestra una asociación entre síntomas depresivos maternos con peor control metabólicos en adolescentes con DM1, incluso al ajustarlo por variable confundentes en el análisis multivariado. Se ha descrito que la depresión materna se correlaciona con menor ajuste psicosocial del adolescente y peor adherencia al tratamiento ${ }^{39}$. El mecanismo se ha intentado explicar en diversos estudios, habiéndose documentado que madres con TDM presentarían un involucramiento excesivo en el manejo de DM1 y en temas no relacionados a la DM1, lo cual en la etapa de la adolescencia resultaría menos beneficioso al no permitir cierta autonomía del joven ${ }^{27,40}$. Esto, sumado a la irritabilidad propia de los síntomas depresivos, generaría mayores conflictos familiares tanto en aspectos generales como relacionados a la DM1 ${ }^{41}$. Otros estudios han demostrado una asociación entre síntomas depresivos maternos y menor monitorización de la adherencia al tratamiento durante la adolescencia ${ }^{25}$.

Durante la adolescencia resulta fundamental una transición armónica desde un involucramiento parental directo en el manejo de la enfermedad a una supervisión por parte de los padres, pero respetando y fomentando la autonomía progresiva que debe asumir el adolescente. Los síntomas depresivos maternos dificultan este proceso desencadenando, ya sea un involucramiento excesivo o una falta de monitorización. Todo lo anterior, se correlacionaría finalmente con menor adherencia al tratamiento, y consecuentemente, peor control metabólico ${ }^{25}$.

En el presente estudio el 17,9\% de los adolescentes presentan síntomas depresivos, prevalencia mayor que adolescentes sin DM1, al comparar con un estudio en adolescentes chilenos que pesquisó síntomas depresivos en $8,3 \%{ }^{42}$. Nuestro estudio no demostró asociación entre síntomas depresivos del adolescente y síntomas depresivos maternos, ni con mal control metabólico, lo cual no concuerda con publicaciones previas donde

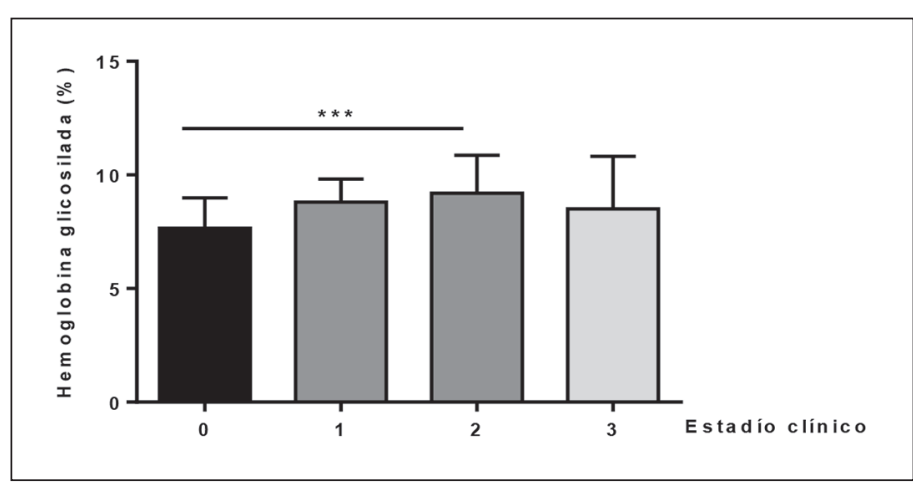

Figura 2. HbA1c promedio según gravedad de síntomas depresivos maternos. 0: Sin síntomas depresivos, 1: Leves, 2: Moderados, 3: Severos. Test de Kruskal-Wallis y post test de Dunn, *** $p<0,001$.

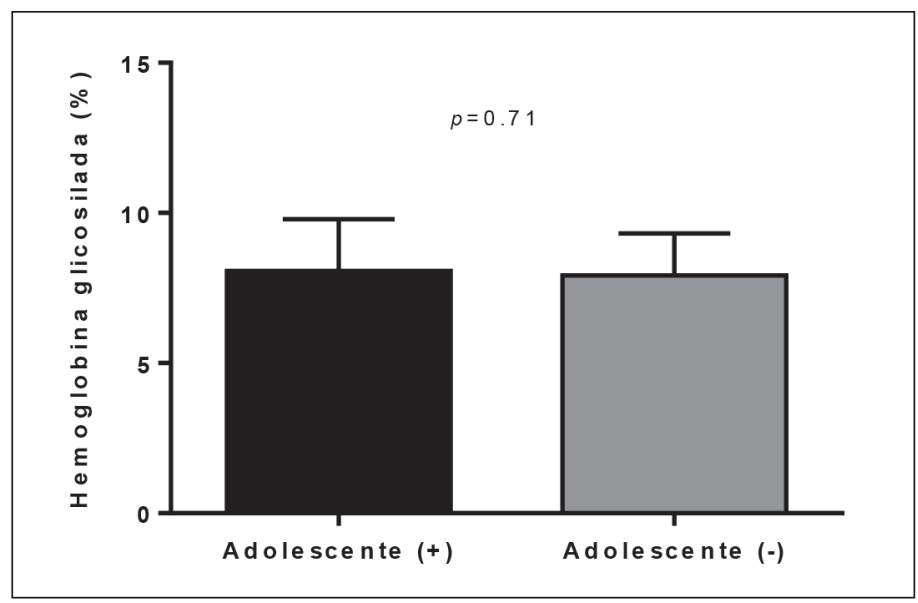

Figura 3. HbA1c promedio en pacientes con (+) y sin (-) síntomas depresivos. Test de U Mann-Whitney.

Tabla 2. OR ajustados significativos para niveles de $\mathrm{HbA} 1 \mathrm{c}>7,5 \%$ (análisis multivariado)

\begin{tabular}{lcc}
\hline Variable & $\begin{array}{c}\text { OR } \\
\text { ajustado }\end{array}$ & $\begin{array}{c}\text { IC } \\
\text { OR ajustado }\end{array}$ \\
\hline Otra enfermedad crónica en adolescente & 3,72 & $1,08-12,85$ \\
Vulnerabilidad en salud según SALUFAM & 5,38 & $1,003-28,95$ \\
Síntomas depresivos maternos & 6,23 & $1,16-33,5$ \\
\hline
\end{tabular}

Variables incluidas en análisis multivariado: sexo, presencia de otra enfermedad crónica en el adolescente, nivel educacional de la madre, presencia de síntomas depresivos maternos y en el adolescente y vulnerabilidad en salud. No se incluyen en tabla aquellas variables que no dieron resultado significativo.

se habría evidenciado una asociación entre estas variables ${ }^{15,18}$. Posiblemente, dado la menor prevalencia de síntomas depresivos en los adolescentes, se necesitaría un estudio con un número mayor de casos, para poder objetivar dicha asociación. 
Probablemente el impacto que tiene el diagnóstico de DM1 sobre la Salud Mental es importante, es por eso que los porcentajes de psicopatología son mayores que la población general, sobre todo durante el primer año desde el diagnóstico ${ }^{43}$. Por esto es fundamental educar y apoyar al paciente y a toda la familia desde el diagnóstico de la enfermedad. Cuando estos síntomas se prolongan en el tiempo se deben pesquisar y manejar oportunamente, dado que se podrían asociar con peor control metabólico, adelantando las complicaciones asociadas a DM1.

Dado las características transversales del estudio no es posible evaluar causalidad, por lo que se debe tener precaución al analizar los datos y sería deseable realizar estudios prospectivos que permitan establecer causalidad, y eventualmente establecer si el reconocimiento y tratamiento de síntomas depresivos maternos mejora el control metabólico. La selección se realizó por conveniencia, por lo que podría existir sesgo de selección, considerando que aquellos pacientes con mala adherencia a controles pudiesen tener síntomas depresivos con mayor frecuencia. También es necesario recalcar que tanto el BDI-II como CDI son métodos de screening, no de diagnóstico de TDM.

\section{Conclusiones}

En este estudio transversal, los síntomas depresivos de las madres se asocian a peor control metabólico de sus hijos adolescentes con DM1. Se hace necesario un enfoque familiar en la atención de adolescentes con DM1, creando programas que realicen intervenciones multidisciplinarias a la familia para disminuir el distrés, mejorar relaciones interpersonales y la manera que enfrentan el proceso de duelo que conlleva el período inicial de la enfermedad. También es fundamental apoyar a los padres en el periodo de transición de la adolescencia que implica un aumento gradual de la autonomía del adolescente, manteniendo algún grado de supervisión hasta que éste pueda hacerse cargo completamente de su enfermedad en la edad adulta ${ }^{44,45}$. Es primordial la creación de programas enfocados en la prevención de psicopatología en padres y adolescentes.

Teniendo en cuenta nuestros resultados y el resto de la bibliografía sobre la alta prevalencia de síntomas depresivos en las madres de adolescentes con DM1, planteamos que sería beneficioso un tamizaje universal de síntomas depresivos tanto en las madres como en los propios adolescentes. Aunque con nuestro trabajo no resulte posible demostrar causalidad, nos parece que pesquisar aquellos pacientes y madres que presentan estos síntomas es fundamental para poder derivarlos de manera oportuna y así poder recibir orientación y tratamiento, lo cual mejorará su calidad de vida y podría redundar en una mejoría del control metabólico.

\section{Responsabilidades Éticas}

Protección de personas y animales: Los autores declaran que los procedimientos seguidos se conformaron a las normas éticas del comité de experimentación humana responsable y de acuerdo con la Asociación Médica Mundial y la Declaración de Helsinki.

Confidencialidad de los datos: Los autores declaran que han seguido los protocolos de su centro de trabajo sobre la publicación de datos de pacientes.

Derecho a la privacidad y consentimiento informado: Los autores han obtenido el consentimiento informado de los pacientes y/o sujetos referidos en el artículo. Este documento obra en poder del autor de correspondencia.

\section{Conflicto de intereses}

Los autores declaran no tener conflicto de intereses.

\section{Financiamiento}

El estudio cuenta con financiamiento del Concurso Becario Residente 2018, Dirección de Investigación y Doctorado, Escuela de Medicina, Pontificia Universidad Católica de Chile.

\section{Agradecimientos}

Agradecemos a los adolescentes y sus madres que participaron. Además, agradecer a todo el equipo tanto médico como no médico (enfermeras, nutricionistas, psicólogas) de los equipos de DM1 de ambos hospitales donde se realizó el estudio. 


\section{Referencias}

1. Patterson CC, Dahlquist GG, Gyürüs E, Green A, Soltész G. Aurodiab Study Group. Incidence trends for childhood type 1 diabetes in Europe during 19892003 and predicted new cases 2005-20: a multicentre prospective registration study. Lancet. 2009;373:2027-33.

2. Carrasco E, Dorman J, Mondragón A, Santos JL. Increasing incidence of type 1 diabetes in population from Santiago of Chile: Trends in a period of 18 years (1986-2003). Diabetes Metab Res Rev. 2006;22:34-37.

3. DCCT/EDIC Research Group. Risk Factors for Cardiovascular Disease in Type 1 Diabetes. Diabetes 2016;65:1370-9.

4. Cengiz E, Xing D, Wong JC, et al. T1D Exchange Clinic Network. Severe hypoglycemia and diabetic ketoacidosis among youth with type 1 diabetes in the T1D Exchange clinic registry. Pediatr Diabetes. 2013;14:447-54.

5. Barnea-Goraly N, Raman M, Mazaika P, et al. Alterations in white matter structure in young children with type 1 diabetes. Diabetes Care. 2014;37:332-40.

6. Salardi S, Porta M, Maltoni G, et al. Ketoacidosis at diagnosis in childhoodonset diabetes and the risk of retinopathy 20 years later. J Diabetes Complications. 2016;30:55-60.

7. Zabeen B, Craig ME, Virk SA, et al. Insulin Pump Therapy is associated with lower rates of retinopathy and peripheral nerve abnormality. Plos One. 2016;11:e0153033.

8. Gubitosi-Klug RA, Sun W, Cleary PA, et al. Effects of prior intensive insulin therapy and risk factors on patientreported visual function outcomes in the Diabetes Control and Complications Trial/Epidemiology of Diabetes Interventions and Complications (DCCT/ EDIC) Cohort. JAMA Ophthalmol. 2016;134:137-45.

9. Wang N, Guo C, Han P, et al Glycated albumin indicates peripheral diabetic neuropathy. Acta Diabetol. 2016;53:973-9.

10. Nathan DM, DCCT/EDIC Research Group. The Diabetes Control and Complications rial/Epidemiology of Diabetes Interventions and Complications Study at 30 Years: Overview. Diabetes Care. 2014;37:9-16.

11. Orchard TJ, Nathan DM, Zinman B, et al. Writing Group for the DCCT/EDIC Research Group. Association between 7 years of intensive treatment of type 1 diabetes and long-term mortality. JAMA. 2015;313:45-53.

12. Miller KM, Foster NC, Beck RW, et al. Current State of Type 1 Diabetes treatment in the U.S.: Updated Data From the T1D Exchange Clinic Registry.
Diabetes Care. 2015; 38: 971-8.

13. Díaz-Cárdenas C, Wong C, Vargas N. Grado de control metabólico en niños y adolescentes con diabetes mellitus tipo 1 . Rev Chil Pediatr. 2016;87:43-7.

14. Cameron F, Garvey K, Hood K, et al. ISPAD Clinical Practice Consensus Guidelines 2018: Diabetes in adolescence. Pediatr Diabetes. 2018; 19 (Suppl. 27):250-61.

15. Whittemore R, Jaser S, Chao A, et al. Psychological experience of parents of children with type 1 diabetes: a systematic mixed-studies review. Diabetes Educ. 2012; 38: 562-79.

16. Butwicka A, Zalepa A, Fendler W, et al. Maternal depressive symptoms predict acute hospitalization among children with type 1. Diabetes. Pediatr Diabetes. 2013;14:288-94.

17. Reynolds KA, Helgeson VS. Children with Diabetes Compared to Peers: Depressed? Distressed?: A Meta-Analytic Review. Ann Behav Med. 2011; 42: 29-41.

18. Kongkaew C, Jampachaisri K, Chaturongkul CA, et al. Depression and adherence to treatment in diabetic children and adolescents: a systematic review and meta-analysis of observational studies. Eur J Pediatr. 2014;173:203-12.

19. Campbell MS, Schatz DA, ChenV, et al. A contrast between children and adolescents with excellent and poor control: the T1D exchange clinic registry experience. Pediatr Diabetes. 2014;15:110-7.

20. Blackman SM, Raghinaru D, Adi S, et al. Insulin pump use in young children in the T1D Exchange clinic registry is associated with lower hemoglobin Alc levels than injection therapy. Pediatr Diabetes. 2014;15:564-72.

21. Miller KM, Beck RW, Bergenstal RM, et al. Evidence of a strong association between frequency of self-monitoring of blood glucose and hemoglobin A1c levels in T1D Exchange clinic registry participants. Diabetes Care 2013;36:200914.

22. Galler A, Bolloe E, Meusers M, et al. Comparison of Glycemic and Metabolic Control in Youth With Type 1 Diabetes With and Without Antipsychotic Medication: Analysis From the Nationwide German/Austrian Diabetes Survey (DPV). Diabetes Care 2015; 38 : 1051-7.

23. Gallegos-Macias AR, Macias SR, Kaufman $\mathrm{E}$, et al. Relationship between glycemic control, ethnicity and socioeconomic status in Hispanic and white nonHispanic youths with type 1 diabetes mellitus. Pediatr Diabetes. 2003;4:19-23.

24. Haugstvedt A, Wentzel-Larsen T, Graue M, et al. Fear of hypoglycaemia in mothers and fathers of children with Type 1 diabetes is associated with poor glycaemic control and parental emotional distress: a population-based study. Diabet Med. 2010;27:72-8.

25. Mackey ER, Struemph K, Powell PW, et al. Maternal Depressive Symptoms and Disease Care Status in Youth with Type 1 Diabetes. Health Psychol. 2014; 33:783-91.

26. Cunningham NR, Vesco AT, Dolan LM, Hood KK. From Caregiver Psychological Distress to Adolescent Glycemic Control: The Mediating Role of Perceived Burden around Diabetes Management. J Pediatr Psychol. 2011;36):196-205.

27. Eckshtain D, Ellis DA, Kolmodin K, et al. The Effects of Parental Depression and Parenting Practices on Depressive Symptoms and Metabolic Control in Urban Youth with Insulin Dependent Diabetes. J Pediatr Psychol. 2010;35:42635.

28. Rumburg T, Lord J, Savin Kimberly, et al. Maternal Diabetes Distress is Linked to Maternal Depressive Symptoms and Adolescents' Glycemic Control. Pediatr Diabetes. 2017;18: 67-70.

29. Ministerio de Salud. Guía Clínica AUGE Diabetes Mellitus tipo 1. Santiago, Minsal 2013.

30. Delamater A, de Wit M, McDarby V, et al. ISPAD Clinical Practice Consensus Guidelines 2018: Psychological care of children and adolescents with type 1 diabetes. Pediatr Diabetes. 2018;19(Suppl. 27):237-49.

31. Chiang JL, Maahs DM, Garvey KC, et al. Type 1 diabetes in children and adolescents: a position statement by the American Diabetes Association. Diabetes Care. 2018;41:2026-44.

32. Melipillan R, Cova F, Rincón P, Valdivia M. Propiedades Psicométricas del Inventario de Depresión de Beck-II en Adolescentes Chilenos. Terapia psicológica. 2008;26:59-69.

33. Beck AT, Steer RA, Brown G. Manual for the Beck Depression Inventory-II (BDIII). The Psychological Corporation. 1996.

34. Puschel K, Repetto P, Solar M, et al. "Diseño y validación del instrumento SALUFAM: un instrumento de valoración de la salud familiar con alto valor predictivo clínico para la atención primaria chilena. Rev Med Chile. 2012;140:417-25.

35. Corathers SD, Kichler J, Jones NH, et al. Improving depression screening for adolescents with type 1 diabetes. Pediatrics. 2013;132:e1395-402.

36. Cáceres C, Collado R. Estandarización del cuestionario de depresión infantil (CDI), versión adaptada de Coggiola y Guillon, a la población escolar de ambos sexos, de 8 a 14 años, del Gran Santiago. Tesis (licenciado en psicología). Universidad Diego Portales, Santiago de Chile: 1994. $105 \mathrm{p}$. 
37. Health Quality Ontario. Point-of-Care Hemoglobin Alc Testing: An EvidenceBased Analysis. Ont Health Technol Assess Ser. 2014;14:1-30.

38. Departamento de Epidemiología. Encuesta Nacional de Salud 2016-2017. Ministerio de Salud, Chile. 2018.

39. Jaser S, Grey M. A pilot study of observed parenting and adjustment in adolescents with type 1 diabetes and their mothers. J Pediatr Psychol. 2010;35:738-47.

40. Wiebe DJ, Gelfand D, Butler JM, et al. Longitudinal associations of maternal depressive symptoms, maternal involvement, and diabetes management across adolescence. J Pediatr Psychol. 2011;36:837-46.

41. Wysocki T, Harris MA, Buckloh LM, et al. Effects of behavioral family systems therapy for diabetes on adolescents' family relationships, treatment adherence, and metabolic control. J Pediatr Psychol. 2006;31:928-38.

42. Vicente B, Saldivia S, de la Barra F, et al. Salud mental infanto-juvenil en Chile y brechas de atención sanitarias. Rev Med Chile 2012;140:447-57.

43. Yi-Frazier JP, Cochrane K, Whitlock
$\mathrm{K}$, et al. Trajectories of Acute DiabetesSpecific Stress in Adolescents With Type 1 Diabetes and Their Caregivers Within the First Year of Diagnosis. J Pediatr Psychol. 2018;43:645-53.

44. Whittemore R, Zincavage RM, Jaser SS, et al. Development of an eHealth Program for Parents of Adolescents With Type 1 Diabetes. Diabetes Educ. 2018;44:72-82.

45. Jaser SS, Lord JH, Savin K, et al.

Developing and Testing an Intervention to Reduce Distress in Mothers of Adolescents with Type 1 Diabetes. Clin Pract Pediatr Psychol. 2018;6:19-30. 